\title{
Mixed-Norm Multi-Objective Robust Controller Applied to a Very Flexible Aircraft
}

\author{
Sohrab Haghighat*, Hugh H.T. Liu ${ }^{\dagger}$ \\ University of Toronto Institute for Aerospace Studies, Toronto, Ontario, M3H 5T6, Canada \\ and \\ Joaquim R. R. A. Martins ${ }^{\ddagger}$ \\ Department of Aerospace Engineering, University of Michigan, 1320 Beal Avenue, Ann Arbor, MI
}

\begin{abstract}
A mixed-norm multi-objective robust controller is designed and integrated with a very flexible aircraft. Due to the aircraft high level of flexibility, the aircraft's aeroservoelastic equations of motion are extremely nonlinear. In a very flexible aircraft, even slight deviation of flight conditions from their nominal values can highly affect the vehicle's stability. A control architecture is proposed to minimize the adverse effects of trim velocity variation on the stability and performance of the closed-loop system. The linear matrix inequalities technique is used to express the control design process as a convex optimization problem. The performance of the designed controller when applied to a very flexible aircraft trimmed at various cruise velocities is evaluated and compared with a non-robust $H_{2}$ controller.
\end{abstract}

\section{Introduction}

During the past decade, high-altitude long-endurance unmanned aircraft have gained considerable attention from aircraft designers. The simultaneous requirement for a lightweight structure and high aerodynamic performance result in high aspect ratio wings that are very flexible. Such flexible wings exhibit unusual aeroelastic behavior compared to conventional aerial vehicles and have been the subject of study for many researchers ${ }^{1 /[3]}$ High level of rigid-elastic interaction and high sensitivity of structural deflections to flight parameters pose significant challenges to control design procedure. The significant dependency of wing deformation on trim conditions, reduces the stability and performance of a control system which is designed for the nominal flight condition.

Due to the significant interaction between the structural dynamics and the rigid-body dynamics, this family of aircraft suffers from marginal stability and can be deformed into uncontrollable configurations. Therefore, aeroservoelastic tailoring is an integral part of the design $4{ }^{455}$ The inclusion of the active load alleviation control system in the design optimization problem along with the more traditional disciplines (such as aerodynamics and structures) can improve the performance of the aircraft beyond the traditional achievable limits. ${ }^{6}$ The generation of a detailed mathematical model that can model structural deformations along with the rigid-body motions for a flexible aircraft is of great importance. However, no mathematical model can accurately represent a dynamic process and the presence of model/parameter uncertainties can affect the controller performance. Traditionally, the main focus of aeroservoelastic synthesis has been either to achieve the required maneuverability, or to avoid structural failure. And when stability robustness is addressed, the main focus is on robust aeroelastic analysis (e.g. guaranteed flutter margin calculation) in the presence of uncertainties, such as complex valued aerodynamic and real valued structural perturbations $\frac{778}{7 n}$ the case of a very flexible aircraft, the trim conditions and specifically the deformed shape of the wing have a significant

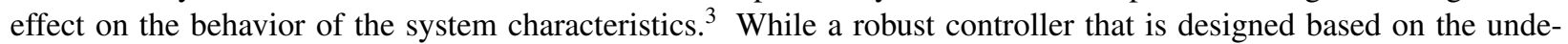
formed shape of the aircraft can handle a slightly flexible aircraft, inaccurate consideration of a highly flexible aircraft wing deformations in control design can lead to instabilities .9

\footnotetext{
*PhD Candidate; sohrab.haghighat@utoronto.ca, University of Toronto Institute for Aerospace Studies, AIAA Member.

${ }^{\dagger}$ Associate Professor; liu@ utias.utoronto.ca, University of Toronto Institute for Aerospace Studies, AIAA Senior Member.

¥Associate Professor; jrram@ umich.edu, Department of Aerospace Engineering, University of Michigan, AIAA Senior Member.
} 
The presence of model inaccuracies and un-modeled dynamics can affect the performance and the stability of the load alleviation controller. As a result, in order to ensure the integrity of the aircraft structures in the face of model uncertainties and at different flight conditions, numerous conditions have to be considered in the design optimization process. Zink et al $!^{10}$ addressed the problem of maneuver load inaccuracies in the design optimization of an active aeroelastic wing and proposed a method to reduce the sensitivity of the optimized structure to the load inaccuracies and reduce the number of the flight conditions considered in the optimization. However the design of the control system was not considered in that work. In another effort, Moulin et al .11 considered the effect of inertial uncertainties due to the inertial deviations of a fighter aircraft wing-tip missile and proposed a robust control framework which was used in a fighter aircraft structural and control design optimization.

The traditional $\mathrm{H}_{2}$ approach attempts to minimize the root-mean-square (rms) value of the performance output driven by unit impulse or unit intensity white noise. The result is a controller adept at handling noises but potentially weak in tracking performance and vulnerable to model uncertainties. The $H_{\infty}$ optimization, on the other hand, minimizes the system output energy to unknown but bounded energy inputs which results in a highly robust system. Therefore, a mixed-norm multi-objective controller can be designed to satisfy both the stability robustness and the temporal performance requirements. Mixed $H_{2} / H_{\infty}$ problems were extensively studied by the control researchers! $\frac{12-14}{14}$ Recent advancements in convex programming have made linear matrix inequalities (LMI) a useful tool for solving a wide variety of control problems especially for multi-objective problems $\frac{15}{16}$ Scherer et al. reported a thorough overview of the application of LMI approach to multi-objective control design!

In this work a mixed $H_{2} / H_{\infty}$ output-feedback controller is presented that is capable of simultaneously handling the rigid-body and the deformable dynamics. This controller is formulated in a two degree of freedom architecture and is represented in linear matrix inequality (LMI) form. The representative LMI problem is solved using CVXOPT convex optimizer. This architecture is specifically developed to improve the controller performance robustness. The mixed-norm controller is designed using the linearized plant that represents the nominal flight condition. A second order uncertainty model is obtained by evaluating the plant dynamics at the neighboring flight conditions. The load alleviation performance of the controller along with its rigid-body regulation capabilities are evaluated when flying through different ' 1 - cosine' gust excitations. The effectiveness of the controller (load alleviation performance) at different flight conditions are compared and it is shown that unlike an $H_{2}$ controller the designed controller maintains the same level of effectiveness at different flight conditions.

In the next section, the nonlinear equations of motion of a very flexible aircraft are presented (Section $\Pi$ ). The linearized equations of motion and control system design are reported in Section III Finally, the simulation results comparing the performance of an $H_{2}$ controller with the proposed mixed $H_{2} / H_{\infty}$ controller are presented in Section IV

\section{Aeroservoelastic Formulation}

The development of a mathematical model that can accurately predict the behavior of a very flexible aircraft is the key in the control design procedure. Some early efforts have tried to obtain the flexible aircraft equations of motion by simply adding a series of second-order differential equations to a rigid-body model where each set of equations represents one structural mode ${ }^{17 \mid 18}$ A more detailed formulation using the mean-axes method is reported by Schmidt et al .1920 The use of aerodynamic strip theory and the assumption that structural displacements are small make the formulation less appealing for our application. Body-fixed axes system has also been used by researchers to develop more sophisticated aircraft models $1 \frac{1,2121}{23}$

The nonlinear equations of motion of a very flexible aircraft derived in this section using a body-fixed axes system. We begin with the Lagrange equations for quasi-coordinates, 


$$
\begin{array}{r}
\frac{\mathrm{d}}{\mathrm{d} t}\left(\frac{\partial L}{\partial \mathbf{V}_{c}}\right)+\tilde{\omega} \frac{\partial L}{\partial \mathbf{V}_{c}}-C_{b i} \frac{\partial L}{\partial \mathbf{R}}=\mathbf{F}, \\
\frac{\mathrm{d}}{\mathrm{d} t}\left(\frac{\partial L}{\partial \boldsymbol{\omega}}\right)+\tilde{V}_{c} \frac{\partial L}{\partial \mathbf{V}_{c}}+\tilde{\omega} \frac{\partial L}{\partial \boldsymbol{\omega}}-D^{T} \frac{\partial L}{\partial \boldsymbol{\theta}}=\mathbf{M}, \\
\frac{\mathrm{d}}{\mathrm{d} t}\left(\frac{\partial \hat{L}}{\partial \mathbf{v}}\right)-\frac{\partial \hat{T}}{\partial \mathbf{u}}+\frac{\partial \hat{\mathcal{F}}}{\partial \mathbf{v}}+\mathcal{L} \mathbf{u}=\hat{U},
\end{array}
$$

where, $\mathcal{F}$ represents the Rayleigh's dissipation term and $(\stackrel{\bullet}{)}$ represents the volume density of the energy terms.Figure 1 shows a deformable aircraft with its original and deformed wing, as well as the associated axes systems. $\mathfrak{F}_{I}$ represents the inertial frame and $\mathfrak{F}_{B}$ the body-fixed frame, not necessarily connected to the center of mass. The absolute position and velocity of an infinitesimal mass element $\mathrm{d} m$ on the aircraft is given by,

$$
\begin{aligned}
& \mathbf{R}=\mathbf{R}_{c}+\mathbf{r}+\mathbf{u} \\
& \mathbf{V}=\dot{\mathbf{R}}=\mathbf{V}_{c}+\boldsymbol{\omega} \times(\mathbf{r}+\mathbf{u})+\dot{\mathbf{u}}
\end{aligned}
$$

The above vectors are graphically represented in Figure 1 .

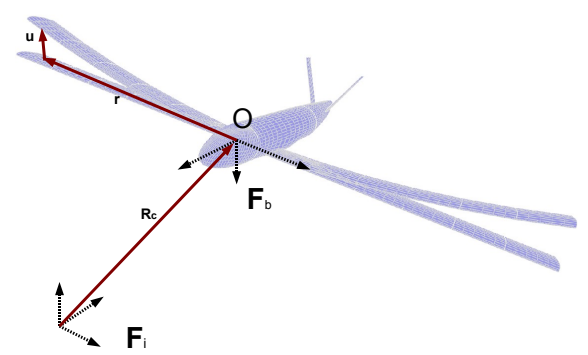

Figure 1. A schematic drawing of a deformed aircraft and the position vectors

The structural deformations are expressed as a linear combination of mode shapes.

$$
\mathbf{u}=\phi_{\mathbf{r}} \boldsymbol{\xi} \Rightarrow \dot{\mathbf{u}}=\phi_{\mathbf{r}} \dot{\boldsymbol{\xi}}
$$

where, $\boldsymbol{\xi}$ is a vector of modal amplitudes and $\phi_{\mathbf{r}}$ represents the modal deflection at location $\mathbf{r}$. Here, the gravitational force is modeled as a distributed force. By using the structural shape functions, Equation (3) can be re-arranged and adapted to the modal form as follows,

$$
\frac{\mathrm{d}}{\mathrm{d} t}\left(\frac{\partial L}{\partial \dot{\boldsymbol{\xi}}}\right)-\frac{\partial T}{\partial \boldsymbol{\xi}}+\frac{\partial \mathcal{U}}{\partial \boldsymbol{\xi}}+\frac{\partial \mathcal{F}}{\partial \dot{\boldsymbol{\xi}}}=\mathbf{f}_{e}
$$

for which, $\mathcal{U}$ and $\mathcal{F}$ represent the strain energy and Rayleigh's dissipation for the complete aircraft structure. Also, $\mathbf{f}_{e}$ represents the generalized modal forces that act on the aircraft structure.

\section{A. Nonlinear Equations of Motion}

The kinetic and potential energies can be expressed in terms of translational and rotational velocities and structural deformations (more detailed derivation of kinetic and potential energy terms can be found in authors' previous work ${ }^{24}$ ). Substituting those terms into Equations (1, 2) and 6) and rearranging them, the equations of motion of a deformable 
aircraft become

$$
\begin{aligned}
& {\left[\begin{array}{ccc}
m \mathcal{I}_{3 \times 3} & X_{1}^{T} & S_{1} \\
X_{1} & J & S_{2}+X_{2} \\
S_{1}^{T} & S_{2}^{T}+X_{2}^{T} & M_{e e}
\end{array}\right]\left\{\begin{array}{l}
\dot{\mathbf{V}}_{c} \\
\dot{\omega} \\
\ddot{\xi}
\end{array}\right\}=} \\
& -\left[\begin{array}{ccc}
m \tilde{\omega} & \tilde{\omega} X_{1}^{T} & 2 \tilde{\omega} S_{1} \\
-\tilde{\omega} X_{1} & \tilde{\omega} J+\dot{J}+\tilde{V}_{c} X_{1} & \tilde{\omega}\left(S_{2}+X_{2}\right) \\
O_{n \times 3} & S_{1}^{T} V_{c}-2 \int_{v} \rho \phi_{\mathbf{r}}{ }^{T} \phi_{\mathbf{r}} \dot{\dot{\xi}} \mathrm{d} v+\int_{v} \rho \phi_{b l r}{ }^{T} \tilde{\omega} \tilde{r}^{T} \mathrm{~d} v & C_{e e}
\end{array}\right]\left\{\begin{array}{l}
\mathbf{V}_{c} \\
\boldsymbol{\omega} \\
\dot{\xi}
\end{array}\right\} \\
& -\left[\begin{array}{cc}
\mathcal{O}_{6 \times 6} & \mathcal{O}_{6 \times n} \\
\mathcal{O}_{n \times 6} & \int_{v} \rho \phi_{\mathbf{r}}^{T} \tilde{\omega}^{2} \phi_{\mathbf{r}} \mathrm{d} v+K_{e e}
\end{array}\right]\left\{\begin{array}{l}
\mathbf{R}_{c} \\
\boldsymbol{\theta} \\
\boldsymbol{\xi}
\end{array}\right\}+\left[\begin{array}{l}
m C_{b i} \\
\mathcal{O}_{3 \times 3} \\
S_{1}^{T} C_{b i}
\end{array}\right]\{\mathbf{g}\}+\left\{\begin{array}{l}
\mathbf{F} \\
\mathbf{M} \\
\mathbf{f}_{e}
\end{array}\right\}
\end{aligned}
$$

where, $\mathbf{F}$ and $\mathbf{M}$ are the total forces and moments about the origin of the body-frame, including propulsive and aerodynamic forces. $\mathbf{f}_{e}$ represents the generalized forces due to elastic deformations and is calculated as,

$$
\mathbf{f}_{e}=\int_{s} \phi_{r}^{T} p \hat{\mathbf{n}} d S,
$$

where $p$ is the aerodynamic pressure acting on the surface of the aircraft. Also, $S_{1}, S_{2}, X_{1}$ and $X_{2}$ are the rigid-elastic interaction terms and are defined as follows,

$$
\begin{aligned}
& S_{1}=\int_{v} \rho \phi_{r} \mathrm{~d} v, \\
& S_{2}=\int_{v} \rho \tilde{r} \phi_{r} \mathrm{~d} v, \\
& X_{1}=\int_{v} \rho \widehat{\phi_{r} \boldsymbol{\xi}} \mathrm{d} v, \\
& X_{2}=\int_{v} \rho \hat{\phi_{r} \boldsymbol{\xi}} \phi_{r} \mathrm{~d} v .
\end{aligned}
$$

As can be seen from Equation (7), the above mentioned terms mainly appear in the inertia matrix and the force vector and create interaction between the rigid and deformable dynamics. As aircraft become more flexible, the relative magnitudes of there terms with respect to the other terms in Equation (7) become larger that results in a closer interaction between the two dynamics.

\section{Aeroservoelastic Control}

The first step in control system development is to extract the linearized nominal plant representation. In order to perform this linearization, the steady-state equations are derived. We start from the equations of motion (7) and set all derivatives and $\boldsymbol{\omega}$ to zero, which yields,

$$
\left\{\begin{array}{l}
\mathbf{F}+m C_{b i} \mathbf{g}=0 \\
\mathbf{M}=0 \\
-K_{e e} \boldsymbol{\xi}+\left(S_{1}\right)^{T} C_{b i} \mathbf{g}+\mathbf{f}_{e}=0
\end{array}\right.
$$

Substituting the perturbed flight parameters into Equation (7) and linearizing, yields,

$$
\begin{aligned}
& \left\{\begin{array}{l}
\delta \dot{\mathbf{V}}_{c} \\
\delta \dot{\boldsymbol{\omega}} \\
\delta \ddot{\boldsymbol{\xi}}
\end{array}\right\}=\mathbf{M}^{-1}\left(\left[\begin{array}{ccc}
\mathcal{O}_{3 \times 3} & m \tilde{V}_{c_{0}} & \mathcal{O}_{3 \times n} \\
\mathcal{O}_{3 \times 3} & \widehat{X_{1_{0}}^{T} V_{c_{0}}}-V_{c_{0}} X_{1_{0}} & \mathcal{O}_{3 \times n} \\
\mathcal{O}_{3 \times 3} & S_{1}^{T} V_{c_{0}} & -C e e
\end{array}\right]\left\{\begin{array}{l}
\delta \mathbf{V}_{c} \\
\delta \boldsymbol{\omega} \\
\delta \dot{\boldsymbol{\xi}}
\end{array}\right\},\right. \\
& \left.+\left[\begin{array}{ccc}
\mathcal{O}_{3 \times 3} & m G & \mathcal{O}_{3 \times n} \\
\mathcal{O}_{3 \times 3} & \mathcal{O}_{3 \times n} & \mathcal{O}_{3 \times n} \\
\mathcal{O}_{3 \times 3} & S_{1}^{T} G & -K_{e e}
\end{array}\right]\left\{\begin{array}{l}
\delta \mathbf{R}_{c} \\
\delta \boldsymbol{\theta} \\
\delta \boldsymbol{\xi}
\end{array}\right\}+\left\{\begin{array}{l}
\delta \mathbf{F} \\
\delta \mathbf{M} \\
\delta \mathbf{f}_{e}
\end{array}\right\}\right)
\end{aligned}
$$

where $M$ and $G$ matrices are as

$$
\mathbf{M}=\left[\begin{array}{ccc}
m & X_{1_{0}}^{T} & S_{1_{0}} \\
X_{1_{0}}^{T} & J_{0} & S_{2_{0}}+X_{2_{0}} \\
S_{1_{0}}^{T} & S_{2_{0}}^{T}+X_{2_{0}}^{T} & M_{e e}
\end{array}\right], \quad G=\left[\begin{array}{ccc}
0 & -g \cos \left(\theta_{0}\right) & 0 \\
-g \cos \left(\theta_{0}\right) & 0 & 0 \\
0 & g \sin \left(\theta_{0}\right) & 0
\end{array}\right] .
$$


From the above equations, it is clear that the linearized equations of motion are highly sensitive to trim condition. Equation (11) can be expressed in a state-space form. The state vector and other system matrices are

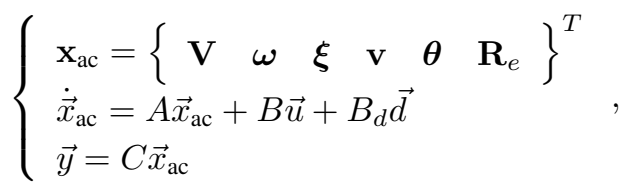

where, $\vec{\theta}$ is the vector of Euler angles $\left(\left[\begin{array}{lll}\phi & \theta & \psi\end{array}\right]^{T}\right)$ and $\vec{v}$ represents structural deflection rates $(\vec{v}=\dot{\xi})$. The $B_{1}$ matrix is used to model effect of disturbances, such as gusts, on the aircraft dynamics. Elevator and aileron deflections are the two control inputs used in this work. The proposed 2-DOF control architecture used in this work is shown in Figure 2

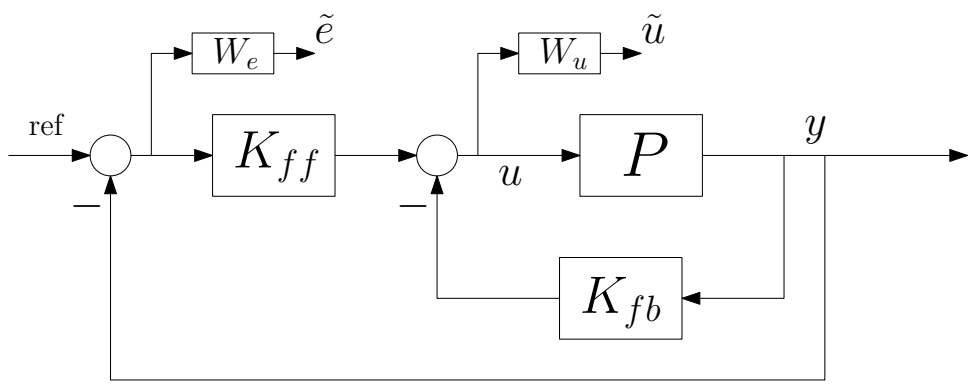

Figure 2. The proposed two DOF control architecture

In this work, a control system is designed to stabilize the plant and improve the performance robustness in the presence of a $20 \%$ flight speed variation. The aerodynamic forces and moments that affect the cruise condition are quadratic functions of the cruise speed. In addition to those, the stability and control derivatives show quadratic dependency on the flight speed. Therefore, a quadratic uncertainty model is used for the control design procedure.

$$
\begin{aligned}
& A=A_{o}+A_{1} \delta_{v}+A_{2} \delta_{v}^{2} \\
& B=B_{o}+B_{1} \delta_{v}+B_{2} \delta_{v}^{2} \\
& B_{d}=B_{d_{o}}+B_{d 1} \delta_{v}+B_{d 2} \delta_{v}^{2}
\end{aligned}
$$

where $\delta_{V}=\frac{V-V_{o}}{0.2 V_{o}}$ and $\delta_{V} \in[-1,1]$. In order to obtain an uncertainty model, the linearized aircraft dynamics is evaluated at two different flight conditions in addition to the nominal one. The linear and quadratic uncertainty terms can be found from the following set of equations,

$$
\left\{\begin{array}{l}
A_{1}+A_{2}=\left.A\right|_{V_{o}+20 \% V_{o}}-\left.A\right|_{V_{o}} \\
0.5 A_{1}+0.25 A_{2}=\left.A\right|_{V_{o}+10 \% V_{o}}-\left.A\right|_{V_{o}}
\end{array}\right.
$$

Similar set of equations can be used to find $B_{1}, B_{2}, B_{d 1}$, and $B_{d 2}$. Based on the above definition, the actual plant can be represented as follows,

$$
\begin{aligned}
& \dot{\vec{x}}_{\mathrm{ac}}=\left(A+\sum_{i=1}^{2} \delta_{v}^{i} A_{i}\right) \vec{x}_{\mathrm{ac}}+\left(B+\sum_{i=1}^{2} \delta_{v}^{i} B_{i}\right) \vec{u}+\left(B_{d}+\sum_{i=1}^{2} \delta_{v}^{i} B_{d i}\right) \vec{d} \\
& y=C \vec{x}_{\mathrm{ac}}
\end{aligned}
$$

The above state-space equation can be re-arranged in the following form.

$$
\dot{\vec{x}}_{\mathrm{ac}}=A \vec{x}_{\mathrm{ac}}+B \vec{u}+B_{d} \vec{d}+\sum_{i=1}^{2} \delta_{v}^{i}\left[\begin{array}{lll}
A_{i} & B_{i} & B_{d i}
\end{array}\right]\left\{\begin{array}{l}
\vec{x}_{\mathrm{ac}} \\
\vec{u} \\
\vec{d}
\end{array}\right\}
$$


Using singular value decomposition (SVD) the above equation can be re-arranged as follows:

$$
\dot{\vec{x}}_{\mathrm{ac}}=A \vec{x}_{\mathrm{ac}}+B \vec{u}+B_{d} \vec{d}+\left[\begin{array}{ll}
B_{\Delta 1} & B_{\Delta 2}
\end{array}\right] \overbrace{\left[\begin{array}{ll}
\delta_{v} \mathcal{I}_{r_{1}} & \\
& \delta_{v}^{2} \mathcal{I}_{r_{2}}
\end{array}\right] \underbrace{\left[\begin{array}{lll}
C_{\Delta_{1}} & D_{\Delta u_{1}} & D_{\Delta d_{1}} \\
C_{\Delta_{2}} & D_{\Delta u_{2}} & D_{\Delta d_{2}}
\end{array}\right]\left\{\begin{array}{l}
\vec{x}_{\mathrm{ac}} \\
\vec{u} \\
\vec{d}
\end{array}\right\}}_{y_{\Delta}}}^{u_{\Delta}}
$$

where $r_{i}=\operatorname{rank}\left(\left[\begin{array}{ccc}A_{i} & B_{i} & B_{d i}\end{array}\right]\right)$. The control system can be represented in linear fractional transformation (LFT) form, where $P$ includes all the frequency-domain weightings (Figure 3 .

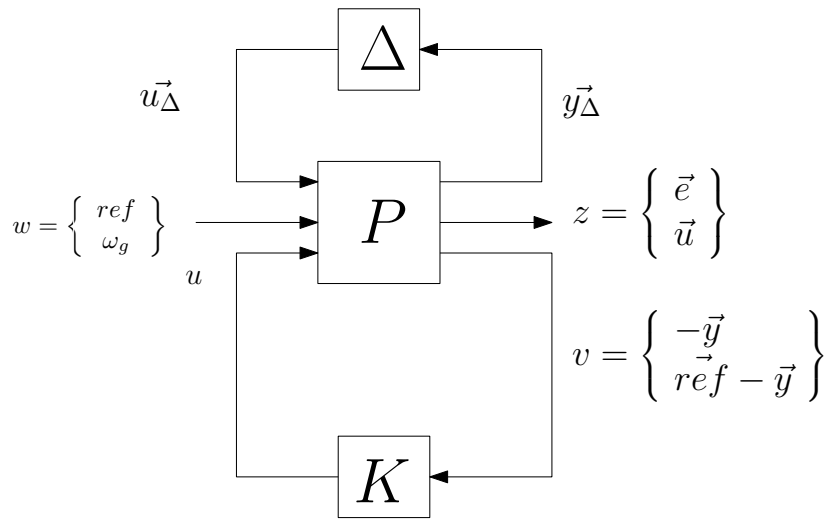

Figure 3. Control system in LFT form

The two feed-back and feed-forward controllers are combined. The gain matrix for both controllers is written as,

$$
K=\left[\begin{array}{ll}
K_{f b} & K_{f f}
\end{array}\right]
$$

The system output is divided into two categories. The performance output $(z)$ represents the tracking (regulating) error and the control surface deflections. In order to obtain good temporal performance, the following linear quadratic cost function is used for minimization.

$$
J=\int_{0}^{\infty}\left(\vec{e}^{T} Q \vec{e}+\vec{u}^{T} R \vec{u}\right) d t=\|z\|_{2}^{2}
$$

The above mentioned cost function can be optimized by minimizing $\left\|T_{z w}\right\|_{2}$ which is a classical $H_{2}$ control design problem. On the other hand, according to small gain theorem, in order to ensure the robust stability of the aircraft in presence of uncertainties, $\left\|T_{y_{\Delta} u_{\Delta}}\right\|_{\infty}$ should be smaller than $\|\Delta\|_{\infty}$. Therefore, a mixed-norm minimization is of interest in this work. Having all elements for control synthesis in place, the design problem can be stated as,

$$
\begin{array}{cc}
\min & \left\|T_{y_{\Delta} u_{\Delta}}\right\|_{\infty}+\rho\left\|T_{z w}\right\|_{2} \\
\text { w.r.t. } & K
\end{array}
$$

The LMI techniques and convex optimization methods are employed to make the problem of finding the controller gains more tractable. More detailed discussion on the LMI representation of $H_{\infty}$ and $H_{2}$ problem can be found in the

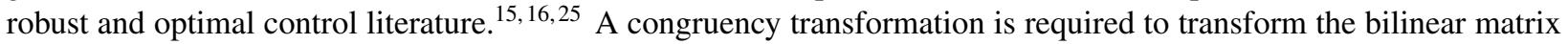
inequalities (BMI) that represent these problems into LMI problems. The resulting LMI representation for the $H_{\infty}$ 
problem is

$$
\begin{aligned}
& \left(\begin{array}{ccc}
\left(A X+B N C_{v}\right)+\left(A X+B N C_{v}\right)^{T} & B_{\Delta} & \left(C_{\Delta} X+D_{\Delta u} N C_{v}\right)^{T} \\
* & -\gamma I & \mathcal{O} \\
* & * & -\gamma I
\end{array}\right)<0, \\
& X>0, \\
& M C_{v}=C_{v} X .
\end{aligned}
$$

And the LMI formulation of the $H_{2}$ problem is

$$
\begin{aligned}
& \left(\begin{array}{cc}
\left(A X+B N C_{v}\right)+\left(A X+B N C_{v}\right)^{T} & B_{1} \\
* & -I
\end{array}\right)<0, \\
& \left(\begin{array}{cc}
X & \left(C_{z} X+D_{z} N C_{v}\right)^{T} \\
* & Q
\end{array}\right)>0, \\
& \operatorname{Tr}(Q)<\alpha, \\
& M C_{v}=C_{v} X \text {. }
\end{aligned}
$$

By using the above-mentioned LMI problems, the control design problem represented in the Equation (21) can be casted as follows,

$$
\begin{array}{cc}
\text { min. } & \gamma+\rho \alpha \\
\text { w.r.t. } & X, N \\
\text { s.t } & \text { Equations 22, 23, 25, 26 and 27 }
\end{array}
$$

While $X$ and $N$ matrices are found by solving the above problem, the $M$ matrix can be found by solving Equation (24). Finally, the controller gain has the following form,

$$
K=N M^{-1} \text {. }
$$

The above LMI problems are casted in semi-definite programming form and $C V X O P T^{26}$ is used to solve the problems to find the appropriate matrices and the controller gains.

\section{Results}

A high altitude long endurance (HALE) UAV with large aspect ratio is the target of this study. The geometry of the baseline aircraft is shown in Figure 4 and the geometrical parameters are listed in Table 1 . More detailed information of this UAV can be found in earlier work.2|22

Table 1. Aircraft geometric properties

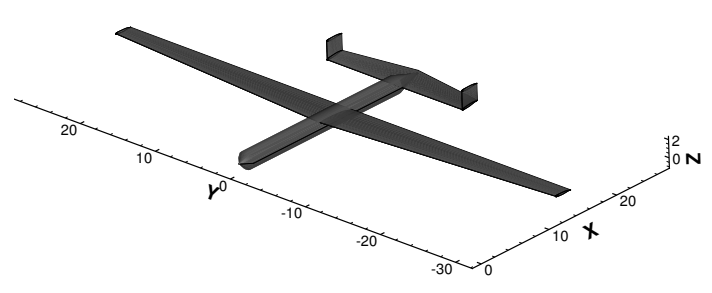

Figure 4. 3D geometry of a generic UAV with a large span

\begin{tabular}{lr}
\hline \hline Property & Value \\
\hline Fuselage length & $26.4 \mathrm{~m}$ \\
Wing span & $58.6 \mathrm{~m}$ \\
Wing area & $196 \mathrm{~m}^{2}$ \\
Wing taper ratio & 0.48 \\
HT span & $18 \mathrm{~m}$ \\
HT area & $53.5 \mathrm{~m}^{2}$ \\
HT taper ratio & 0.7 \\
VT span & $4 \mathrm{~m}$ \\
VT area & $8.9 \mathrm{~m}^{2}$ \\
VT taper ratio & 0.81 \\
\hline
\end{tabular}


A NACA 4415 airfoil is used for the wing cross-section and the chord-wise location of the spar is set to $45 \%$ of the chord. The nominal cruise speed is set to $80 \mathrm{~m} / \mathrm{s}$. A mixed-norm $H_{2} / H_{\infty}$ controller and an $H_{2}$ controller are designed. Two different flight conditions are analyzed: an altitude change maneuver where the aircraft is required to climb from $2,000 \mathrm{~m}$ to $2,500 \mathrm{~m}$ in $40 \mathrm{~s}$ (Figures 5, 8), and flying through atmospheric turbulence where a ' 1 - cosine' profile of the following form is used for gust simulation.

$$
w_{g}=\frac{\bar{w}_{g}}{2}\left(1-\cos \frac{2 \pi t}{L_{g}}\right),
$$

where $\bar{w}_{g}$ is the maximum gust velocity, and $L_{g}$ is the gust length. For our purposes, $\bar{w}_{g}$ is set to $15 \mathrm{fps}(4.575 \mathrm{~m} / \mathrm{s})$ and value of $0.25 \mathrm{~s}$ is used for $L_{g}$. The gust scale factor $\left(L_{g}\right)$ is selected so that it has the maximum excitation on the aircraft structure is achieved (Figures 90 12).

Both controllers have good performance when applied to the nominal plant. However, the application of the $\mathrm{H}_{2}$ controller for gust load alleviation to the low speed trimmed aircraft resulted in excessive structural vibration that destabilized the aircraft. On the other hand, the mixed-norm controller managed to stabilize all three plants without significant performance degradation. The application of $\mathrm{H}_{2}$ controller for altitude gain maneuver also resulted in aircraft instabilities. In this case, however, the high speed trimmed aircraft experienced aileron saturation and excessive oscillations. The mixed-norm controller successfully stabilized the aircraft at all three cruise speeds. The low speed trimmed aircraft showed a slower climb rate and did not reach the desired altitude in $40 \mathrm{~s}$. Based on the obtained results it can be concluded that aircraft flying at lower speeds than the nominal design speed are more vulnerable to atmospheric turbulence. Also, when flying at higher speeds than the nominal flight condition the higher than required control action can induce structural oscillation that results in aileron saturation which is used for structural load alleviation. However, when the plant uncertainties are used in the control design procedure, the designed controller can successfully stabilize the aircraft in a wider range of flight conditions.

In addition to these two flight conditions, the load alleviation performance of the two controllers are evaluated at 5 different cruise speeds with the nominal flight speed set to $80 \mathrm{~m} / \mathrm{s}$. Three different gust lengths of 20, 40, and 80 meters are considered for this analysis. The results of this comparison is presented in Table 2 The average maximum stress $\left(\sigma_{\max }\right)$ and the variation bound are presented here. When the proposed mixed norm controller was used the average $\sigma_{\max }$ was lower for all three gust lengths. Even more important than lower average $\sigma_{\max }$ is the stress variation bound which have been smaller when a mixed norm controller was applied. This smaller variation bound results in a structural design which is less sensitive to the flight condition variations.

Table 2. Load alleviation capabilities (mixed-norm versus $H_{2}$ controller)

\begin{tabular}{lcccc}
\hline \hline & \multicolumn{2}{c}{$H_{2}$ controller } & \multicolumn{2}{c}{ Mixed-norm controller } \\
\hline Gust length $(\mathrm{m})$ & Nominal $\sigma_{\max }(\mathrm{MPa})$ & Variation bound $(\%)$ & Nominal $\sigma_{\max }(\mathrm{MPa})$ & Variation bound (\%) \\
\hline 20 & 262 & 5.34 & 259 & 3.86 \\
40 & 274 & 3.28 & 268 & 2.23 \\
80 & 292 & 4.1 & 282 & 3.54 \\
\hline
\end{tabular}

\section{Conclusions}

A 2-DOF mixed-norm control architecture that is capable of performing altitude tracking and load alleviation was presented in this work. The control structure was specifically designed to improve the stability robustness of a very flexible aircraft when subjected to trim conditions variation. The uncertainty model is obtained by trimming the aircraft at different cruise speeds and calculating the variation in the aircraft state-space matrices. The optimal control problem was casted in LMI form where convex optimization techniques can be used to solve the control problem very efficiently. The performance of the designed mixed-norm controller and an $\mathrm{H}_{2}$ controller are evaluated by performing gust load alleviation and climb maneuver simulations. While both controllers achieved desirable performance with the nominal plant, the $\mathrm{H}_{2}$ controller destabilized the aircraft when it was not trimmed at the nominal cruise speed. On the other hand, the designed $H_{2} / H_{\infty}$ controller managed to stabilize the aircraft at different flight speeds and achieve desirable performance. 


\section{References}

${ }^{1}$ Patil, M. J. and Hodges, D. H., "Flight dynamics of Highly Flexible Flying Wings," Journal of Aircraft, Vol. 43, No. 3, 2006 , pp. $1790-1798$.

${ }^{2}$ Shearer, C. M. and Cesnik, C. E., "Nonlinear Flight Dynamics of Very Flexible Aircraft," Journal of Aircraft, Vol. 44, 2007, pp. 1528 -1545.

${ }^{3}$ Frulla, G., Cestino, E., and Marzocca, P., "Critical Behaviour of Slender Wing Configurations," Proceedings of the Institution of Mechanical Engineers, Part G: Journal of Aerospace Engineering, Vol. 224, No. 5, May 2010, pp. 587-600.

${ }^{4}$ Livne, E., "Future of Airplane Aeroelasticity," Journal of Aircraft, Vol. 40, 2003, pp. 1066-1092.

${ }^{5}$ Noll, T. E., Ishmael, S. D., Henwood, B., Perez-Davis, M. E., Tiffany, G. C., Madura, J., Gaier, M., Brown, J. M., and Wierzbanowski, T., "Technical Findings, Lessons Learned, and Recommendations Resulting from the Helios Prototype Vehicle Mishap," Tech. Rep. 20070022260, NASA, 2007.

${ }^{6}$ Haghighat, S., Liu, H. H. T., and Martins, J. R. R. A., “Aeroservoelastic Design Optimization of a Flexible Wing,” Journal of Aircraft, 2011, Submitted.

${ }^{7}$ Chavez, F. R. and Schimdt, D. K., "Systems Approach to Characterizing Aircraft Aeroelastic Model Variation for Robust Control Applications," AIAA Guidance, Navigation and Control Conference, Montreal, Canada, August 2001.

${ }^{8}$ Borglund, D. and Ringertz, U., "Solution of Flutter Eigenvalue Problem with Mixed Structural/Aerodynamic Uncertainty," Journal of Aircraft, Vol. 48, No. 1, 2011, pp. 343-348.

${ }^{9}$ Haghighat, S., Liu, H. H. T., and Martins, J. R. R. A., "Application of Model Predictive Control to Gust Loads Alleviation Systems," AIAA Atmospheric Flight Mechanics, Chicago, AIAA 2009 - 5929, 2009.

${ }^{10}$ Zink, S., Raveh, D., and Mavris, D., "Robust Structural Design of an Active Aeroelastic Wing with Maneuver Load Inaccuracies," Journal of Aircraft, Vol. 41, No. 3, 2004, pp. 585-593.

${ }^{11}$ Moulin, B., Idan, M., and Karpel, M., "Aeroservoelastic Structural and Control Optimization Using Robust Design Schemes," Journal of Guidance,Control, and Dynamics, Vol. 25, No. 1, 2002, pp. 152-159.

${ }^{12}$ Khargonekar, P. P. and Rotea, M. A., "Mixed $\mathrm{H}_{2} / H_{\infty}$ Control: A Convex Optimization Approach," IEEE Transactions On Automatic Control, Vol. 36, No. 7, July 1991, pp. 824-837.

${ }^{13}$ Zhou, K., Glover, K., and Doyle, J., "Mixed $H_{2}$ and $H_{\infty}$ Performance Objectives I: Robust Performance Analysis," IEEE Transaction on Automatic Control, Vol. 39, No. 8, August 1994, pp. 1564-1574.

${ }^{14}$ Doyle, J., Zhou, K., and Glover, K., "Mixed $H_{2}$ and $H_{\infty}$ Performance Objectives II: Optimal Control," IEEE Transaction on Automatic Control, Vol. 39, No. 8, August 1994, pp. 1575-1587.

${ }^{15}$ Boyd, S., Ghaoui, L. E., Feron, E., and Balakrishnan, V., Linear Matrix Inequalities in System and Control Theory, Vol. 15 of Studies in Applied Mathematics, SIAM, 1994.

${ }^{16}$ Scherer, C., Gahinet, P., and Chilali, M., "Multiobjective Output-Feedback Control via LMI Optimization," IEEE Transaction on Automatic Control, Vol. 42, No. 7, July 1997, pp. 896-911.

${ }^{17}$ McLean, D. and Prasad, R. A., "A Structural Load Alleviation Control System for a Large Aircraft," Transactions of the Institute of Measurement and Control, Vol. 2, No. 1, Jan. - Mar. 1980, pp. 25-37.

${ }^{18}$ Aouf, N., Boulet, B., and Botez, R., "Robust Gust Load Alleviation for a Flexible Aircraft," Canadian Aeronautics and Space Journal, Vol. 46, No. 3, September 2000, pp. 131-140.

${ }^{19}$ Waszak, M. R. and Schmidt, D. K., "Flight Dynamics of Aeroelastic Vehicles," Journal of Aircraft, Vol. 25, No. 6, 1988 , pp. 563-571.

${ }^{20}$ Schmidt, D. K. and Raney, D. L., "Modeling and Simulation of Flexible Flight Vehicles," Journal of Guidance, Control and Dynamics, Vol. 24, No. 3, 2001, pp. 539-546.

${ }^{21}$ Meirovitch, L. and Tuzcu, I., "Integrated Approach to the Dynamics and Control of Maneuvering Flexible Aircraft,” Tech. Rep. CR-2003211748, NASA, June 2003.

${ }^{22}$ Shearer, C. M. and Cesnik, C. E. S., "Trajectory Control for Very Flexible Aircraft," Journal of Guidance, Control, and Dynamics, Vol. 31 , No. 2, 2008, pp. 340-357.

${ }^{23} \mathrm{Su}$, W. and Cesnik, C. E. S., "Nonlinear Aeroelasticity of a Very Flexible Blended-Wing-Body Aircraft," Journal of Aircraft, Vol. 47, No. 5, 2010, pp. 1539-1553.

${ }^{24}$ Haghighat, S., Liu, H. H. T., and Martins, J. R. R. A., "Application of Robust Control Design Techniques to the Aeroservoelastic Design Optimization of a Very Flexible UAV Wing," Proceedings of the 13th AIAA/ISSMO Multidisciplinary Analysis Optimization Conference, Fort Worth, TX, September 2010.

${ }^{25}$ Lee, C., Salapaka, S. M., and Voulgaris, P. G., "Two Degree of Freedom Robust Optimal Control Design using A Linear Matrix Inequality Optimization," Joint 48th IEEE Conference on Decision and Control and 28th Chinese Control Conference, Shanghai, China, December 2009 , pp. 714-719.

${ }^{26}$ Dahl, J. and Vandenberghe, L., CVXOPT, September 2010, http://abel.ee.ucla.edu/cvxopt/. 


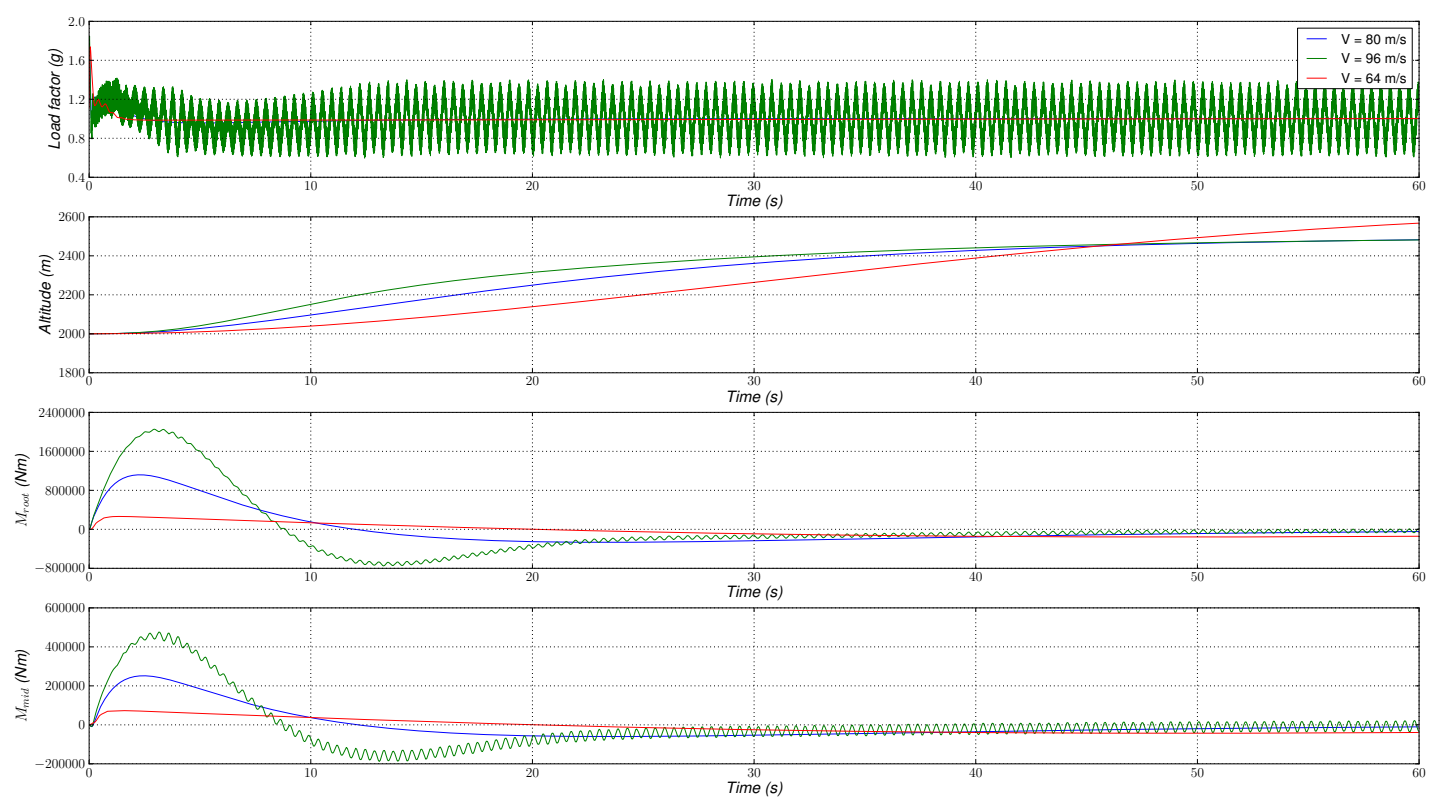

Figure 5. Altitude, load factor and wing bending moment (altitude gain with an $\mathrm{H}_{2}$ controller)
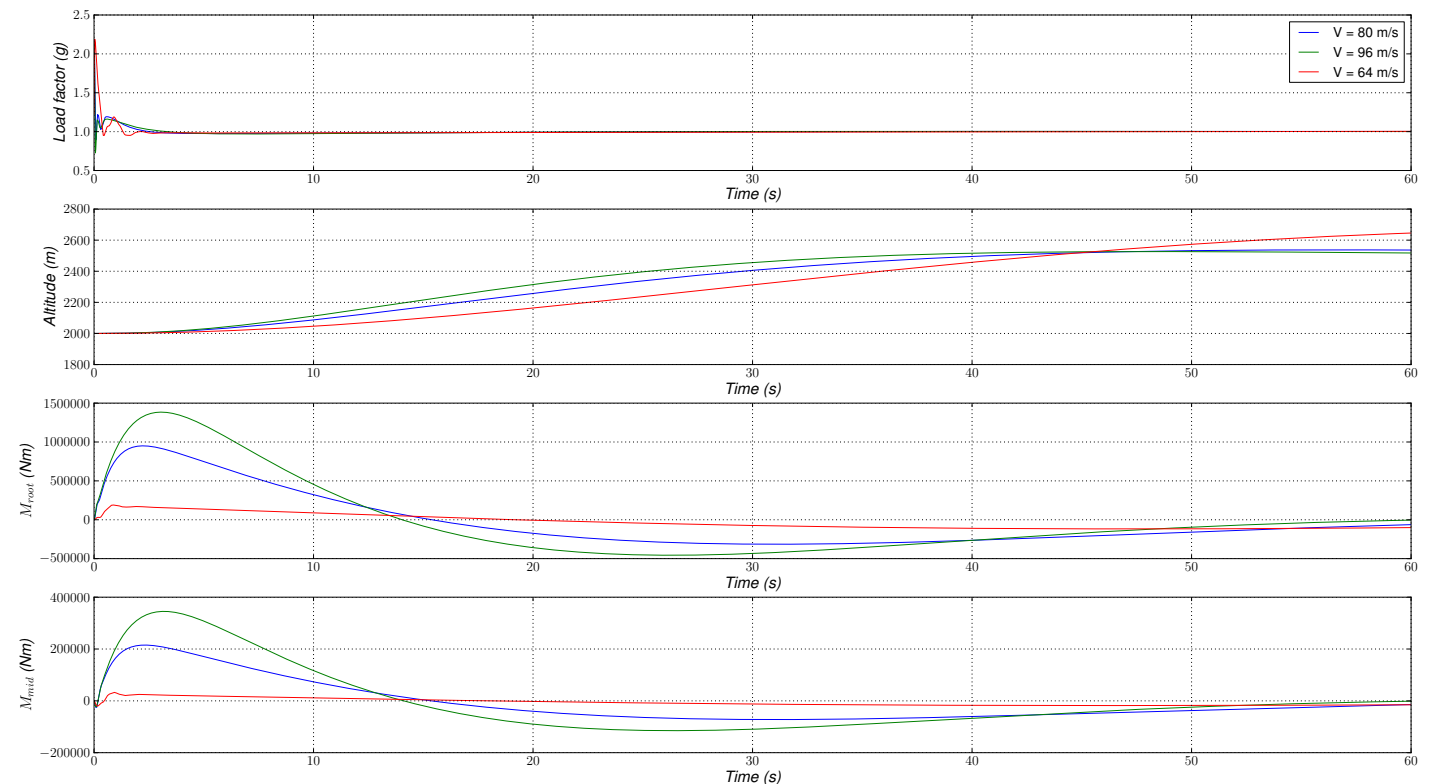

Figure 6. Altitude, load factor and wing bending moment (altitude gain with a mixed $\mathrm{H}_{2} / \mathrm{H}_{\infty}$ controller) 

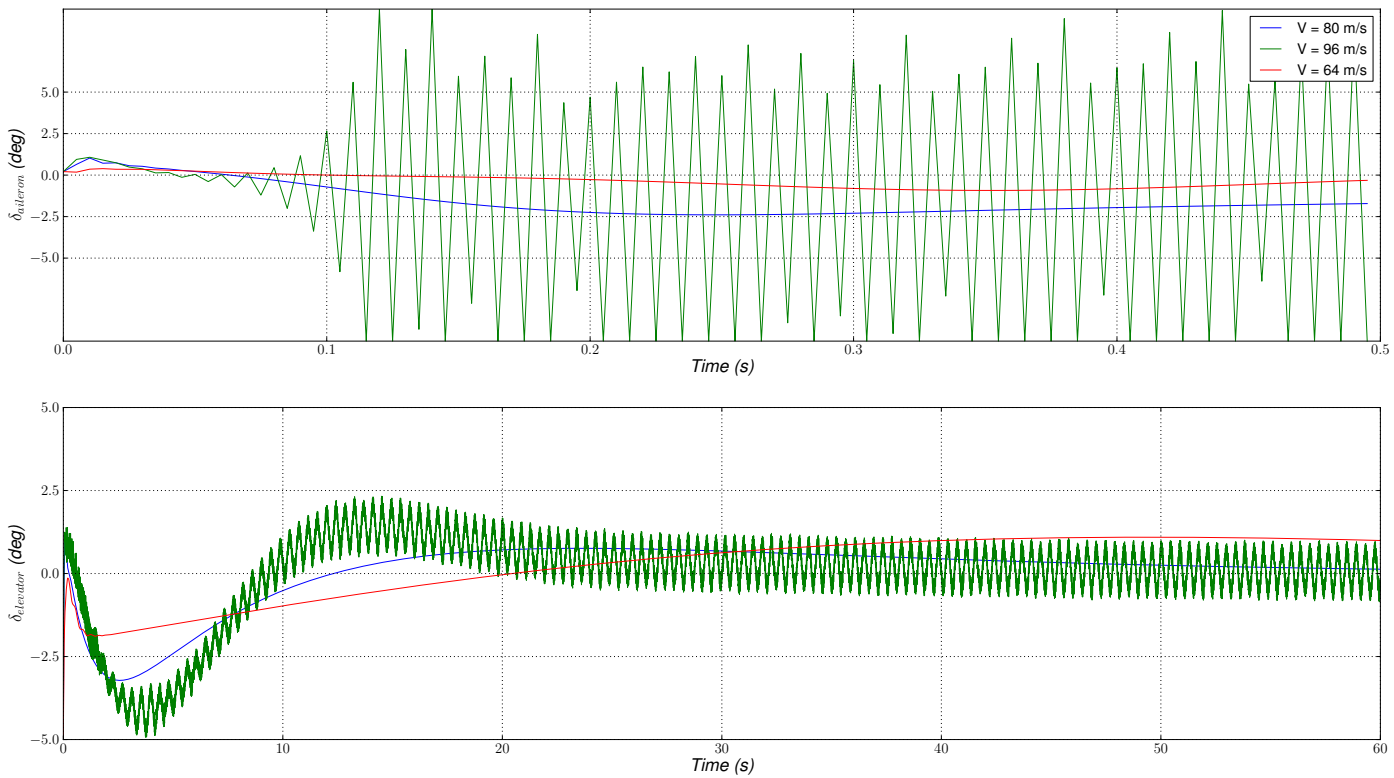

Figure 7. Control surface deflections (altitude gain with an $H_{2}$ controller)
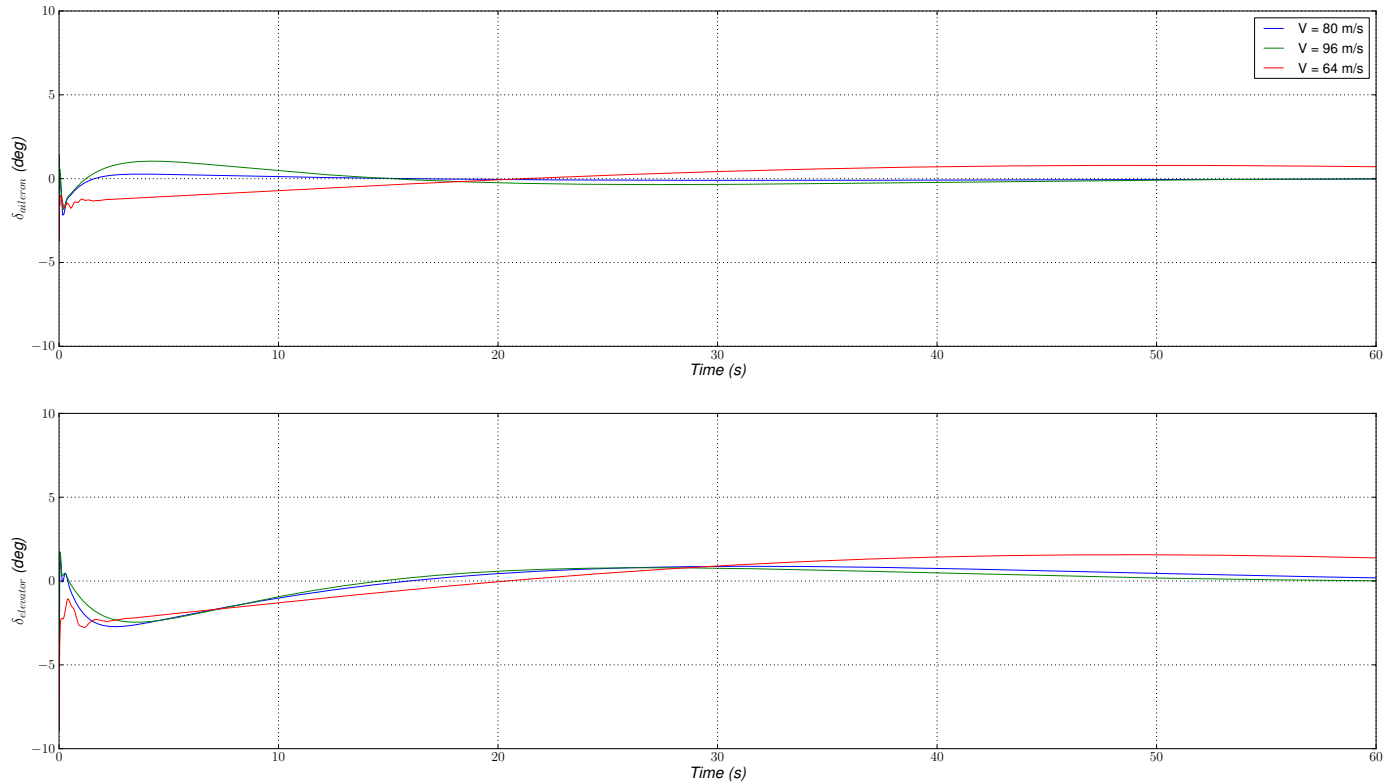

Figure 8. Control surface deflections (altitude gain with a mixed $H_{2} / H_{\infty}$ controller) 

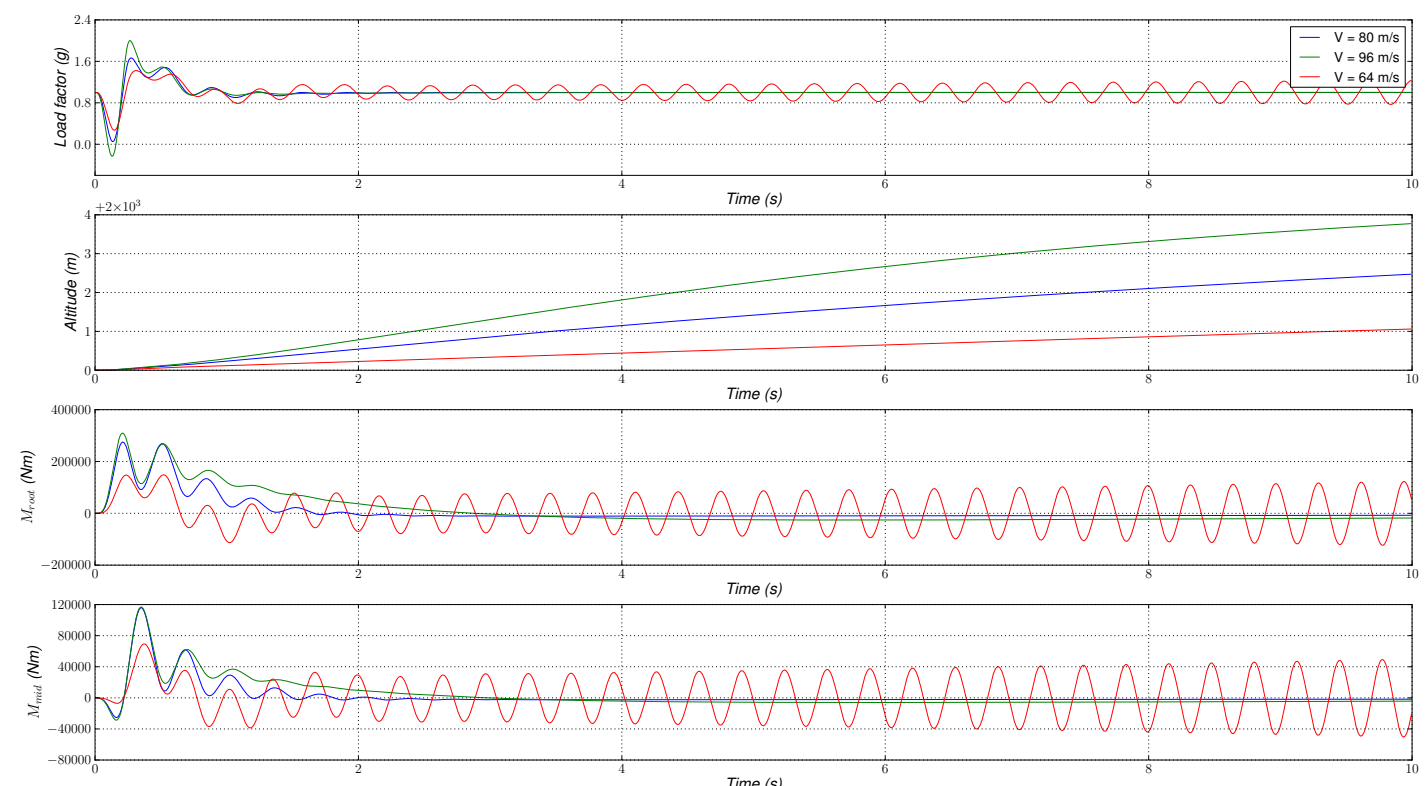

Figure 9. Altitude, load factor and wing bending moment (' 1 - cosine' gust with an $H_{2}$ controller)
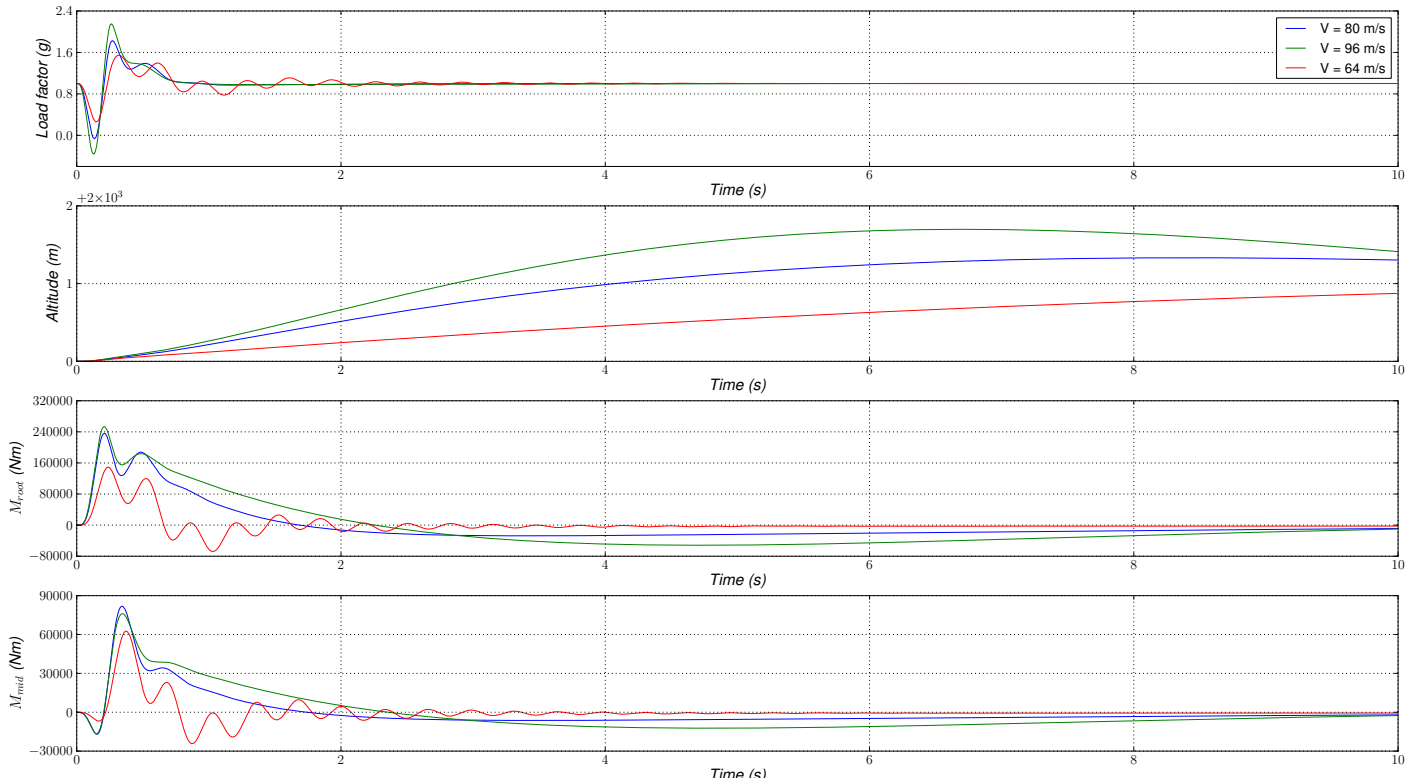

Figure 10. Altitude, load factor and wing bending moment (' 1 - cosine’ gust with a mixed $H_{2} / H_{\infty}$ controller) 

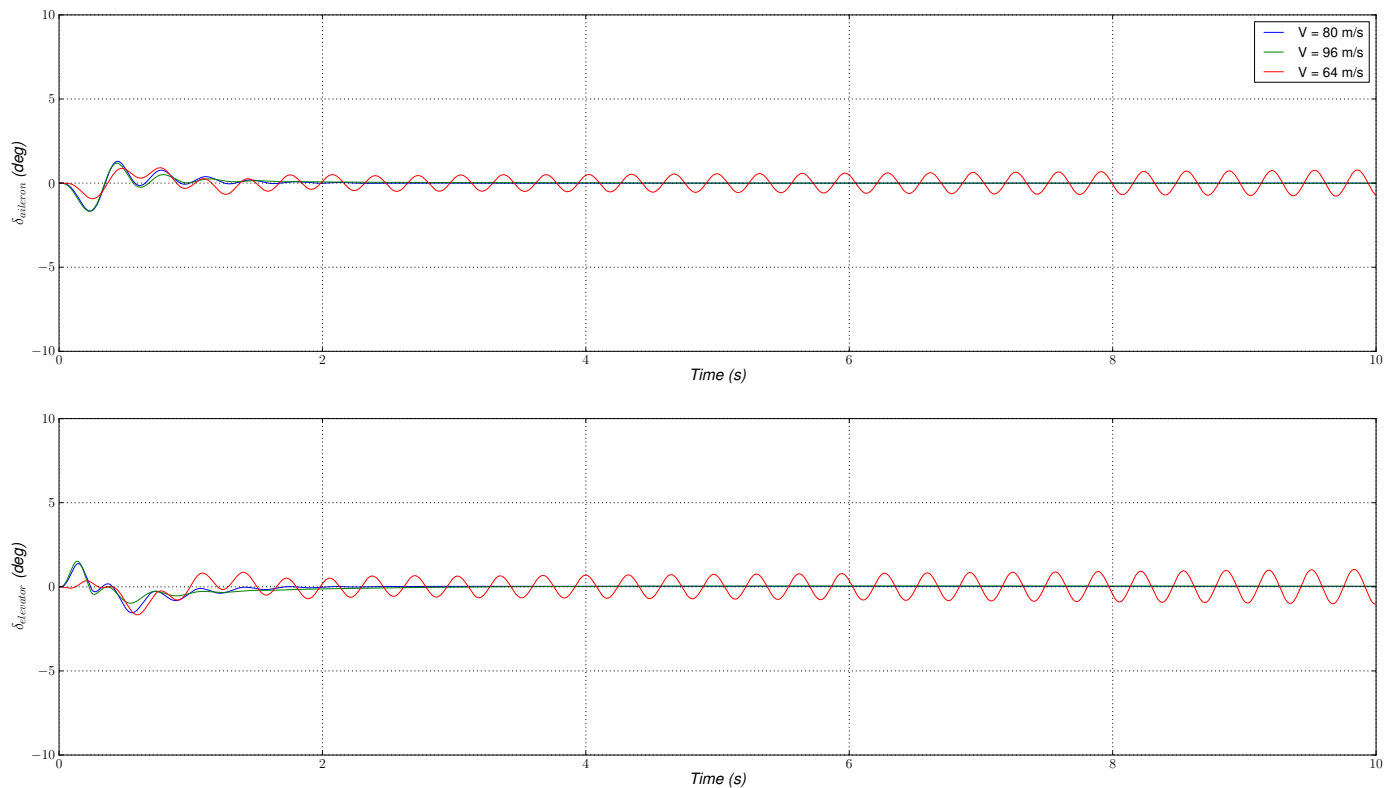

Figure 11. Control surface deflections (' 1 - cosine’ gust with an $H_{2}$ controller)
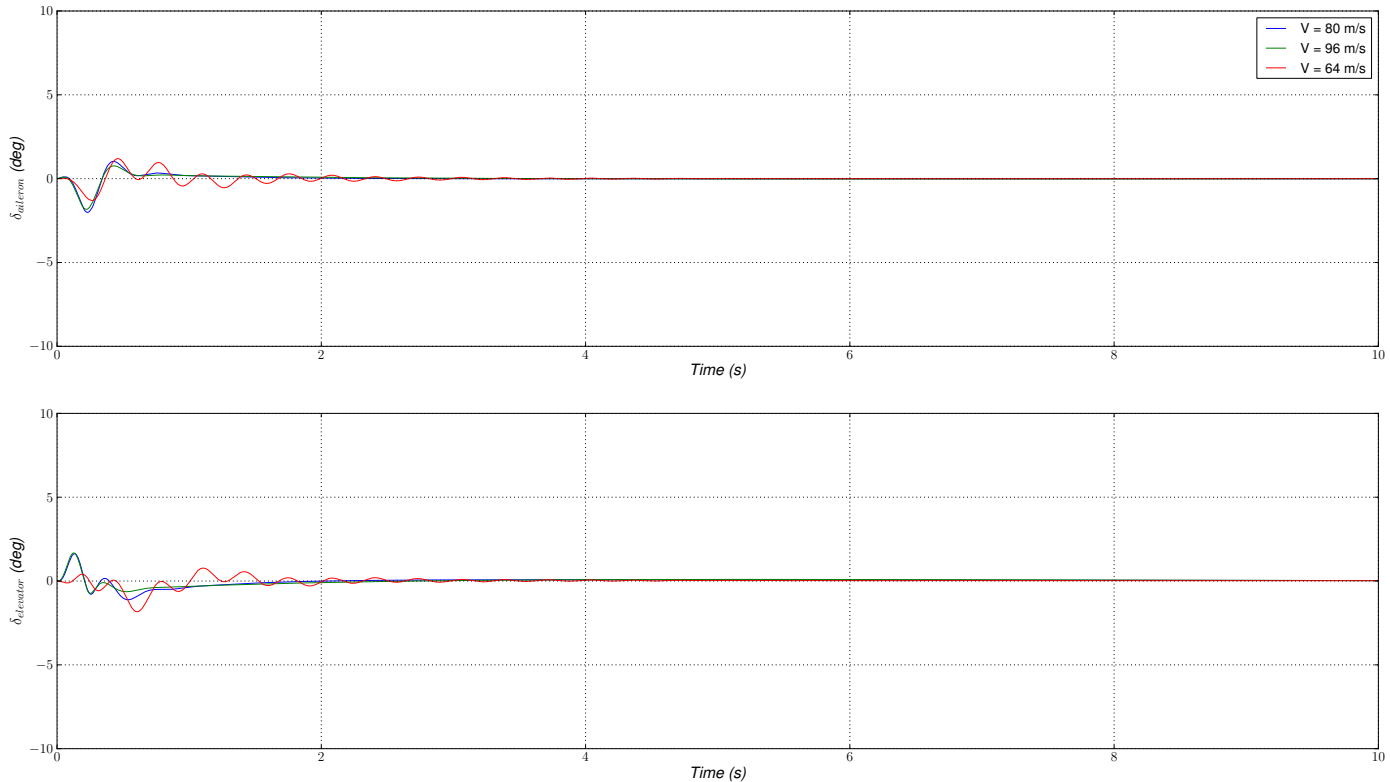

Figure 12. Control surface deflections (' 1 - cosine' gust with a mixed $H_{2} / H_{\infty}$ controller) 